Edinburgh Preprint: 93/526

Southampton Preprint SHEP 92/93-24

PACS numbers: 12.38.G, 14.40

\title{
Quenched Heavy-Light Decay Constants
}

\section{UKQCD Collaboration}

\author{
R.M. Baxter, S.P. Booth, K.C. Bowler, S. Collins, D.S. Henty, R.D. Kenway, \\ D.G. Richards, H.P. Shanahan, J.N. Simone, A.D. Simpson, B.E. Wilkes \\ Department of Physics, The University of Edinburgh, Edinburgh EH9 3JZ, Scotland
}

A.K. Ewing, L. Lellouch, C.T. Sachrajda, H. Wittig Physics Department, The University, Southampton SO9 5NH, UK

\begin{abstract}
We present results for heavy-light decay constants, using both propagating quarks and the static approximation, in $O(a)$-improved, quenched lattice QCD. At $\beta=6.2$ on a $24^{3} \times 48$ lattice we find $f_{D}=185+4$ (stat) $\begin{aligned} & +42 \\ & -3\end{aligned}$ (syst) $\mathrm{MeV}, f_{B}=160 \begin{aligned} & +6 \\ & -6\end{aligned}-53 \mathrm{MeV}$, $f_{D_{s}} / f_{D}=1.18_{-2}^{+2}$ and $f_{B_{s}} / f_{B}=1.22_{-3}^{+4}$, in good agreement with earlier studies. From the static theory we obtain $f_{B}^{\text {stat }}=253 \begin{array}{ll}+16 & +105 \\ -15 & -14\end{array} \mathrm{MeV}$. We also present results from a simulation at $\beta=6.0$ on a $16^{3} \times 48$ lattice, which are consistent with those at $\beta=6.2$. In order to study the effects of improvement, we present a direct comparison of the results using both the Wilson and the improved action at $\beta=6.0$.
\end{abstract}




\section{Introduction}

The leptonic decay constants, $f_{P}$, of pseudoscalar mesons composed of a heavy and a light quark play an important rôle in weak-interaction phenomenology. In particular $f_{B}$, or more strictly $f_{B} \sqrt{B_{B}}$ (where $B_{B}$, the $B$ parameter of $B^{0}-\bar{B}^{0}$ mixing, is expected to be close to one), is one of the principal unknown quantities needed for the determination of the $C P$-violating phase in the Standard Model, as well as other properties of weak decays. Lattice QCD offers the opportunity for a non-perturbative computation of the operator matrix elements which are necessary for the determination of the decay constants and $B$ parameters.

During the last few years there have been several lattice computations of the decay constants of "heavy-light" pseudoscalar (and vector) mesons. The results for the decay constant of the $D$ meson, obtained using the Wilson action for the quarks, are in the region of $200 \mathrm{MeV}$ (using a normalisation for which $f_{\pi}=132 \mathrm{MeV}$ ). For example, in his 1989 review S. Sharpe quoted [1] $f_{D} \simeq 180 \pm 25$ (stat) \pm 30 (syst) $\mathrm{MeV}$ as his summary of the lattice results. More recent simulations with Wilson fermions also give results in this range [2]-[5]. The experimental bound is $f_{D}<290 \mathrm{MeV}$ [

In the heavy-quark limit the scaling law for the decay constant of a heavy-light pseudoscalar meson is $f_{P} \sqrt{M_{P}} \sim$ constant (up to mild logarithmic corrections). Lattice simulations using heavy-quark masses in the charm region indicate that there are large corrections to this scaling law (of order $40 \%$ at the charm quark mass, decreasing to about $15 \%$ at the mass of the bottom quark) [2, 3, [4]. The value of the decay constant of the $B$ meson deduced from these simulations is in the region of $180 \mathrm{MeV}$. The conclusion that there are violations of the scaling law is supported by the large value for $f_{P} \sqrt{M_{P}}$ deduced from simulations obtained using a static (i.e infinitely-massive) heavy quark [5, 0, 8, 9, 10, 11, 12].

The important results and conclusions quoted above were obtained from simulations in which the mass of the heavy quark is large in lattice units (up to about a half). One may therefore worry that discretisation errors significantly contaminate the results. In this paper we present the results for decay constants of heavy-light mesons computed using the $O(a)$-improved lattice action proposed by Sheikholeslami and Wohlert [13, with which the discretisation errors in operator matrix elements (and hence in the computed values of the decay constants) can be reduced from $O\left(m_{Q} a\right)$ to $O\left(\alpha_{s} m_{Q} a\right)$, where $m_{Q}$ is the mass of the heavy quark [14]. This formal reduction in discretisation errors provides an important check on the stability of results and conclusions obtained with Wilson fermions. 
The results presented in this paper were obtained from two simulations of quenched QCD, using the Sheikholeslami-Wohlert (SW) or "clover" fermion action for the quarks (see Subsection 1.1 below). Our main results come from a simulation on a $24^{3} \times 48$ lattice at $\beta=6.2$, for which 60 gauge field configurations were generated. Details of this simulation and the determination of the values of the Wilson hopping parameter corresponding to the chiral limit, $\kappa_{\text {crit }}$, and to the mass of the strange quark have been presented in ref. [15]. The heavy quarks have masses in the region of the charm quark mass and we study the behaviour of the decay constants with the mass of the heavy quark. Interpolating to the mass of the charm quark itself, and extrapolating the results to the mass of the $b$ quark, we find that our best results for the decay constants of the $B$ and $D$ mesons are

$$
\begin{aligned}
& f_{D}=185_{-3}^{+4} \text { (stat) }{ }_{-7}^{+42} \text { (syst) } \mathrm{MeV} \\
& f_{B}=160 \begin{array}{ll}
+6 & +53 \\
-6 & -19
\end{array} \mathrm{MeV} \\
& \frac{f_{D_{s}}}{f_{D}}=1.18_{-2}^{+2} \\
& \frac{f_{B_{s}}}{f_{B}}=1.22_{-3}^{+4} \text {. }
\end{aligned}
$$

The details of this calculation and a complete set of results are presented in Section 2 .

The second simulation is on a $16^{3} \times 48$ lattice at $\beta=6.0$, using 36 configurations. The results, which are consistent with those mentioned above, are presented in Section 3. In order to study the effects of improvement on the calculation of heavy-light decay constants, we have repeated the computation for both the Wilson and SW actions using a subset of 16 of these configurations. The results and a discussion are presented in Subsection 3.2.

We have also computed $f_{B}$ in the static approximation (in which contributions of $O\left(1 / m_{b}\right)$ are neglected). A discussion of the calculation and of the results is presented in Section 4 . The result from the simulation at $\beta=6.2$, on 20 of the 60 configurations, is

$$
f_{B}^{\text {stat }}=253 \underset{-15}{+16}(\text { stat }) \stackrel{+105}{+} \text { (syst) } \mathrm{MeV},
$$

and the result at $\beta=6.0$ on all 36 configurations is

$$
f_{B}^{\text {stat }}=286 \begin{array}{rr}
+8 & +67 \\
-10 & -42
\end{array}
$$

Finally, Section 5 contains our conclusions. 


\subsection{Improved Action and Operators}

The SW action is

$$
S_{F}^{S W}=S_{F}^{W}-i \frac{\kappa}{2} \sum_{x, \mu, \nu} \bar{q}(x) F_{\mu \nu}(x) \sigma_{\mu \nu} q(x),
$$

where $S_{F}^{W}$ is the Wilson action:

$$
S_{F}^{W}=\sum_{x}\left\{\bar{q}(x) q(x)-\kappa \sum_{\mu}\left[\bar{q}(x)\left(1-\gamma_{\mu}\right) U_{\mu}(x) q(x+\hat{\mu})+\bar{q}(x+\hat{\mu})\left(1+\gamma_{\mu}\right) U_{\mu}^{\dagger}(x) q(x)\right]\right\} .
$$

The decay constants of heavy-light pseudoscalar and vector mesons are computed using lattice axial and vector currents as interpolating operators. In order to obtain $O(a)$-improved matrix elements we use "rotated" operators [14]:

$$
\bar{Q}(x)\left(1+\frac{1}{2} \gamma \cdot \overleftarrow{D}\right) \Gamma\left(1-\frac{1}{2} \gamma \cdot \vec{D}\right) q(x)
$$

where $\Gamma$ is one of the Dirac matrices (either $\gamma^{\mu} \gamma^{5}$ or $\gamma^{\mu}$ ), and $Q$ and $q$ represent the fields of the heavy and light quark respectively.

In the static effective theory, in which the heavy propagator is expressed in terms of the link variables [16], it is sufficient to rotate the light-quark fields only [17], i.e. to use the operators

$$
\bar{Q}(x) \Gamma\left(1-\frac{1}{2} \gamma \cdot \vec{D}\right) q(x),
$$

in order to eliminate the $O(a)$-discretisation errors.

\subsection{Renormalisation Constants $Z_{V}$ and $Z_{A}$}

In order to determine the physical values of the decay constants from those obtained in lattice simulations using the interpolating operators in eq. (9), it is necessary to know the corresponding renormalisation constants. These are defined by requiring that $Z_{A} A_{\mu}^{\text {latt }}$ and $Z_{V} V_{\mu}^{\text {latt }}$ are the correctly normalised currents, where the superscript "latt" denotes that the operator is a lattice current. These renormalisation constants have been calculated at one-loop order in perturbation theory for the SW action with rotated operators [18]:

$$
\begin{aligned}
& Z_{V}=1-0.10 g^{2} \\
& Z_{A}=1-0.02 g^{2} .
\end{aligned}
$$

In this paper they are evaluated using the "boosted" coupling suggested in ref. [19]; specifi-

cally, we use $g^{2}=6 /\left(\beta u_{0}^{4}\right)$, where $u_{0}$ is a measure of the average link variable, for which we 
take $u_{0}=\left(8 \kappa_{\text {crit }}\right)^{-1}$. It has been suggested [19, 20 that the use of such an effective coupling, rather than the bare lattice coupling, resums some of the large higher-order corrections, and in particular some of the tadpole diagrams. Using the measured values of $\kappa_{\text {crit }}$ from our simulations, we obtain $Z_{A} \simeq 0.97$ (0.96) and $Z_{V} \simeq 0.83$ (0.82) for the simulation at $\beta=6.2$ (6.0).

In a recent non-perturbative determination of these renormalisation constants, obtained by requiring that the correctly-normalised currents obey the continuum Ward Identities, it was found that $Z_{V}=0.824(2)$ and $Z_{A}=1.09(3)$ [21]. These results were obtained from a simulation at $\beta=6.0$ for one value of the quark mass. It remains to be checked that the results are independent of the quark mass and insensitive to small variations in $\beta$. For this reason, we use the perturbative values, given above, throughout the paper. We note, however, that the non-perturbative value of $Z_{A}$ may be larger by about $15 \%$. In ref. [15] we obtained $f_{\pi} / m_{\rho}=0.138_{-9}^{+6}$, using the perturbative value of $Z_{A}$. A larger value of $Z_{A}$, such as $Z_{A}=1.09$, would bring this result closer to the physical value of 0.172 . However, we also observed that $f_{K} / f_{\pi}$, which does not require $Z_{A}$, was in very good agreement with the experimental value, and therefore we quote values for the ratios $f_{D} / f_{\pi}$ and $f_{B} / f_{\pi}$ in the following sections.

The normalisation of the axial current in the static effective theory is discussed in Section 4 .

\subsection{Error Estimation}

Statistical errors are obtained from a bootstrap procedure [22]. This involves the creation of 1000 bootstrap samples from the original set of $N$ configurations by randomly selecting $N$ configurations per sample (with replacement). Correlators are fitted for each bootstrap sample by minimising $\chi^{2}$. During the fits, correlations among different timeslices are taken into account, whereas correlations among different values of the quark mass are neglected. The latter correlations are preserved by using the same sequence of bootstrap samples at each quark mass. When extrapolating our results to the chiral limit and physical meson masses, the correlation matrix for the fitted quantities is estimated from the full bootstrap ensemble. All quoted statistical errors are obtained from the central $68 \%$ of the corresponding bootstrap distributions 23].

We attempt to quantify the systematic error arising from the uncertainty in the value of the lattice spacing, $a$, determined from properties of light hadrons, and from the string ten- 
sion [15]. The differences between results obtained using our central value for $a^{-1}[\mathrm{GeV}]$ and our upper and lower estimates are quoted as systematic uncertainties in the final estimates for decay constants in physical units. Hereafter, where we quote two errors, the first is statistical and the second is systematic.

\subsection{Extended Interpolating Operators}

In order to isolate the ground state in correlation functions effectively, it is useful to use extended (or "smeared") interpolating operators for the mesons. In particular, in the static theory it has been found to be essential to use smeared operators in order to obtain any signal for the ground state [24]. In this study we use gauge-invariant Jacobi smearing on the heavy-quark field (described in detail in ref. [25]), in which the smeared field, $Q^{S}(\vec{x}, t)$, is defined by

$$
Q^{S}(\vec{x}, t) \equiv \sum_{\vec{x}^{\prime}} J\left(x, x^{\prime}\right) Q\left(\vec{x}^{\prime}, t\right)
$$

where

$$
J\left(x, x^{\prime}\right)=\sum_{n=0}^{N} \kappa_{S}^{n} \Delta^{n}\left(x, x^{\prime}\right)
$$

and

$$
\Delta\left(x, x^{\prime}\right)=\sum_{i=1}^{3}\left\{\delta_{\vec{x}^{\prime}, \vec{x}-\hat{\imath}} U_{i}^{\dagger}(\vec{x}-\hat{\imath}, t)+\delta_{\vec{x}^{\prime}, \vec{x}+\hat{\imath}} U_{i}(\vec{x}, t)\right\} .
$$

Wuppertal smearing [26], which uses the operator $\left(1-\kappa_{S} \Delta\right)^{-1}$ as the kernel of the smearing, corresponds to $N=\infty$, provided that $\kappa_{S}$ is sufficiently small to guarantee convergence. Following the discussion in ref. [25], we choose $\kappa_{S}=0.25$ and use the parameter $N$ to control the smearing radius, defined by

$$
r^{2} \equiv \frac{\sum_{\vec{x}}|\vec{x}|^{2}|J(x, 0)|^{2}}{\sum_{\vec{x}}|J(x, 0)|^{2}}
$$

The values of $N$ and $r$ used in each of the calculations below will be quoted in the corresponding sections.

\section{Decay Constants from the Simulation at $\beta=6.2$}

In this section we present the results obtained for the decay constants of heavy-light mesons from our simulation on 60 configurations of a $24^{3} \times 48$ at $\beta=6.2$, using the SW action in the 
quenched approximation. The computations are performed for four different values of the mass of the heavy quark, corresponding to $\kappa_{h}=0.121,0.125,0.129$ and 0.133 , and for three values of the mass of the light quark, corresponding to $\kappa_{l}=0.14144,0.14226$ and 0.14262 . The mass of the charm quark corresponds approximately to $\kappa_{h}=0.129$. The value of the hopping parameter corresponding to the mass of the strange quark is $\kappa_{s}=0.1419+1$ and the critical value is $\kappa_{\text {crit }}=0.14315+2$ [15.

The decay constants are determined by computing two-point correlation functions of the form

$$
C_{J_{1} J_{2}}^{Q R}(t)=\sum_{\vec{x}}\left\langle 0\left|J_{1}^{Q}(x) J_{2}^{\dagger R}(0)\right| 0\right\rangle
$$

where $J_{1}$ and $J_{2}^{\dagger}$ are interpolating operators which can annihilate or create the pseudoscalar or vector meson being studied. The labels $Q, R$ denote whether a local $(L)$ or smeared $(S)$ interpolating operator is being used. In this simulation we use Jacobi smearing with $N=75$, corresponding to a smearing radius of $r=5.2$. The decay constants are obtained from the matrix elements of the local operators, which are determined by computing both the $C^{S S}$ and $C^{L S}$ correlation functions.

In order to determine the decay constant, it is necessary to know the value of the lattice spacing in physical units. This can be done by relating the lattice measurements of some dimensionful quantity to its physical value, e.g. the mass of a light hadron or $f_{\pi}$. Among the other frequently-used choices are the string tension, $\sqrt{K}$, and the $1 P-1 S$ mass splitting in charmonium. Using $m_{\rho}$ to set the scale in our study of light hadrons [15] we found $a^{-1}\left(m_{\rho}\right)=2.7(1) \mathrm{GeV}$, and a mass spectrum in physical units which was close to experimental values. Furthermore, our determination of the string tension [23] gave $a^{-1}=2.73(5) \mathrm{GeV}$. Encouraged by the consistency of these results, we use

$$
a^{-1}=2.7 \mathrm{GeV}
$$

However, the study described in ref. [15] showed that the measurement of the pion decay constant gave a higher value for the scale, i.e. $a^{-1}\left(f_{\pi}\right)=3.4_{-1}^{+2} \mathrm{GeV}$, using the perturbative value for $Z_{A}$. In order to get an estimate of the systematic uncertainties in the final numbers, we evaluate all our results using the central value of $a^{-1}\left(f_{\pi}\right)$ as well, and quote the difference as the upper systematic error on decay constants. The lower systematic error is obtained from the uncertainty of $-0.1 \mathrm{GeV}$ in $a^{-1}\left(m_{\rho}\right)$.

In an attempt to reduce the systematic errors associated with the value of the renormalisation 
constant of the axial current, we also compute $f_{D, B} / f_{\pi}$, and determine $f_{D, B}$ by using the physical value of $f_{\pi}$.

\subsection{Decay Constants of Pseudoscalar Mesons}

In order to determine the pseudoscalar decay constants, we start by fitting the two-point correlation function

$$
\begin{aligned}
C_{P P}^{S S}(t) & \equiv \sum_{\vec{x}}\left\langle 0\left|P^{S}(\vec{x}, t) P^{\dagger S}(0)\right| 0\right\rangle \\
& \rightarrow \frac{Z_{P S}^{2}}{2 M_{P}} \exp \left(-M_{P} L_{t} / 2\right) \cosh \left(M_{P}\left(L_{t} / 2-t\right)\right)
\end{aligned}
$$

where $P$ is the pseudoscalar density, $Z_{P^{S}}=\left\langle 0\left|P^{S}(0)\right| P\right\rangle$ and $L_{t}$ is the temporal extent of the lattice. This correlation function gives the best determination of the masses of the heavy-light pseudoscalars. Symmetrizing in Euclidean time, the fitting range was chosen to be $13 \leq t \leq 22$ for all three values of the light-quark mass. Good plateaus in the effective mass were observed and stable fits obtained. The values of the masses of the pseudoscalar and vector mesons for the twelve $\kappa_{h}-\kappa_{l}$ combinations are presented in Table 11. The values obtained by linear extrapolation to the chiral limit for the light quark are also tabulated.

At large values of $t$, the ratio of correlation functions

$$
\frac{C_{A P}^{L S}(t)}{C_{P P}^{S S}(t)} \rightarrow \frac{\left\langle 0\left|A_{4}^{L}(0)\right| P\right\rangle}{\left\langle 0\left|P^{S}(0)\right| P\right\rangle} \tanh \left(M_{P}\left(L_{t} / 2-t\right)\right)
$$

is used to extract the pseudoscalar decay constant, where $A_{4}$ is the temporal component of the axial current. The ratio is fitted in the range $15 \leq t \leq 22$ with the pseudoscalar mass $M_{P}$ (in each bootstrap sample) constrained to its value extracted from fits to eq. (19). In Fig. 11 we plot the ratio of correlators together with the fit to eq. (20) as a function of $t$. Using the value of $Z_{P^{S}}$ obtained from the fits of eq. (19), the matrix elements of the local axial current are obtained. Although there are other ways of determining these matrix elements, we find that the ratios in eq. (20) give the most precise determination.

In Table 2 we present the results for the decay constants (in lattice units) of the pseudoscalar mesons for the twelve $\kappa_{h}-\kappa_{l}$ combinations, as well as the values obtained by linearly extrapolating the results to the chiral limit. We also tabulate the results for the quantity $f_{P} \sqrt{M_{P}}$ which, in the heavy-quark limit, is independent of the mass of the heavy quark (except for a mild logarithmic dependence). 


\begin{tabular}{|c|c|c|c|}
\hline$\kappa_{h}$ & $\kappa_{l}$ & $M_{P}$ & $M_{V}$ \\
\hline \multirow{4}{*}{0.121} & 0.14144 & $0.924+3$ & $0.944+4$ \\
\hline & 0.14226 & $0.900+3$ & $0.920+4$ \\
\hline & 0.14262 & $0.890+4$ & $0.909+5$ \\
\hline & $\kappa_{\text {crit }}$ & $0.875+4$ & $0.894+6$ \\
\hline \multirow{4}{*}{0.125} & 0.14144 & $0.822_{-1}^{+3}$ & $0.847_{-2}^{+4}$ \\
\hline & 0.14226 & $0.799+3$ & $0.823+4$ \\
\hline & 0.14262 & $\begin{array}{l}0.789+4 \\
-2\end{array}$ & $0.811+5$ \\
\hline & $\kappa_{\text {crit }}$ & $\begin{array}{r}0.773+5 \\
-2 \\
\end{array}$ & $\begin{array}{r}0.797+6 \\
-4 \\
\end{array}$ \\
\hline \multirow{4}{*}{0.129} & 0.14144 & $\begin{array}{l}0.715_{-1}^{+3} \\
\end{array}$ & $0.745_{-2}^{+4}$ \\
\hline & 0.14226 & $0.691+3$ & $0.721+4$ \\
\hline & 0.14262 & $0.681+4$ & $0.711+5$ \\
\hline & $\kappa_{\text {crit }}$ & $0.665_{-2}^{+5}$ & $0.695_{-4}^{+6}$ \\
\hline \multirow{4}{*}{0.133} & 0.14144 & $\begin{array}{l}0.599+3 \\
-1\end{array}$ & $\begin{array}{l}0.637_{-2}^{+3} \\
-2\end{array}$ \\
\hline & 0.14226 & $0.574+3$ & $0.613_{-3}^{+4}$ \\
\hline & 0.14262 & $\begin{array}{l}0.564+4 \\
-2\end{array}$ & $0.603+5$ \\
\hline & $\kappa_{\text {crit }}$ & $0.546_{-2}^{+4}$ & $0.588_{-4}^{+6}$ \\
\hline
\end{tabular}

Table 1: Masses (in lattice units) of the pseudoscalar and vector mesons for the twelve $\kappa_{h^{-}} \kappa_{l}$ combinations at $\beta=6.2$ on a $24^{3} \times 48$ lattice. Also presented are the values obtained by linear extrapolation to the chiral limit $\left(\kappa_{l} \rightarrow \kappa_{\text {crit }}=0.14315\right)$. 


\begin{tabular}{|c|c|c|c|c|}
\hline$\kappa_{h}$ & $\kappa_{l}$ & $f_{P} / Z_{A}$ & $f_{P} \sqrt{M_{P}} / Z_{A}$ & $1 /\left(f_{V} Z_{V}\right)$ \\
\hline \multirow{4}{*}{0.121} & 0.14144 & $\begin{array}{l}0.086+1 \\
-1\end{array}$ & $\begin{array}{l}0.083+2 \\
-1\end{array}$ & $\begin{array}{l}0.124+2 \\
-3\end{array}$ \\
\hline & 0.14226 & $0.079+1$ & $0.075+2$ & $0.116+3$ \\
\hline & 0.14262 & $0.076+2$ & $0.071+2$ & $0.111+3$ \\
\hline & $\kappa_{\text {crit }}$ & $\begin{array}{l}0.071+2 \\
-1\end{array}$ & $0.066+2$ & $0.105+4$ \\
\hline \multirow{5}{*}{0.125} & 0.14144 & $0.086+1$ & $0.078+1$ & $0.141+3$ \\
\hline & & $\begin{array}{r}-1 \\
-\end{array}$ & $\begin{array}{r}-1 \\
+\end{array}$ & $\begin{array}{r}-3 \\
-3\end{array}$ \\
\hline & 0.14226 & $0.079_{-1}^{+1}$ & $0.070_{-1}^{+1}$ & $\begin{array}{l}0.132+3 \\
-3\end{array}$ \\
\hline & 0.14262 & $\begin{array}{r}0.076+2 \\
-2\end{array}$ & $\begin{aligned} & 0.067+2 \\
&-2\end{aligned}$ & $0.128+4$ \\
\hline & $\kappa_{\text {crit }}$ & $0.071+2$ & $0.062 \begin{array}{l}+2 \\
-1\end{array}$ & $0.123+4$ \\
\hline \multirow{4}{*}{0.129} & 0.14144 & $\begin{array}{l}0.085+1 \\
-1\end{array}$ & $\begin{array}{l}0.071+1 \\
-1\end{array}$ & 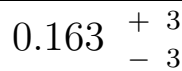 \\
\hline & 0.14226 & $0.078+1$ & $0.065+1$ & $0.155+3$ \\
\hline & 0.14262 & $0.075+2$ & $0.062+1$ & $0.151+4$ \\
\hline & $\kappa_{\text {crit }}$ & $\begin{array}{l}0.071+2 \\
-1\end{array}$ & $0.057+2$ & $0.146_{-5}^{+5}$ \\
\hline \multirow{4}{*}{0.133} & 0.14144 & $\begin{array}{l}20.082+1 \\
-1\end{array}$ & $\begin{array}{l}0.063+1 \\
-1\end{array}$ & $0.193+3$ \\
\hline & 0.14226 & 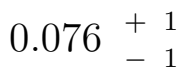 & 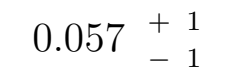 & $0.186+3$ \\
\hline & 0.14262 & $0.073_{-1}^{+1}$ & $0.055+1$ & $0.183+5$ \\
\hline & $\kappa_{\text {crit }}$ & 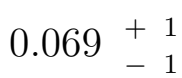 & 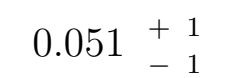 & $0.179+5$ \\
\hline
\end{tabular}

Table 2: The decay constants (in lattice units) of the pseudoscalar and vector mesons. Also shown are the results for the combination $f_{P} \sqrt{M_{P}}$ which in the heavy-quark limit is independent of the heavy-quark mass (up to mild logarithmic corrections). 


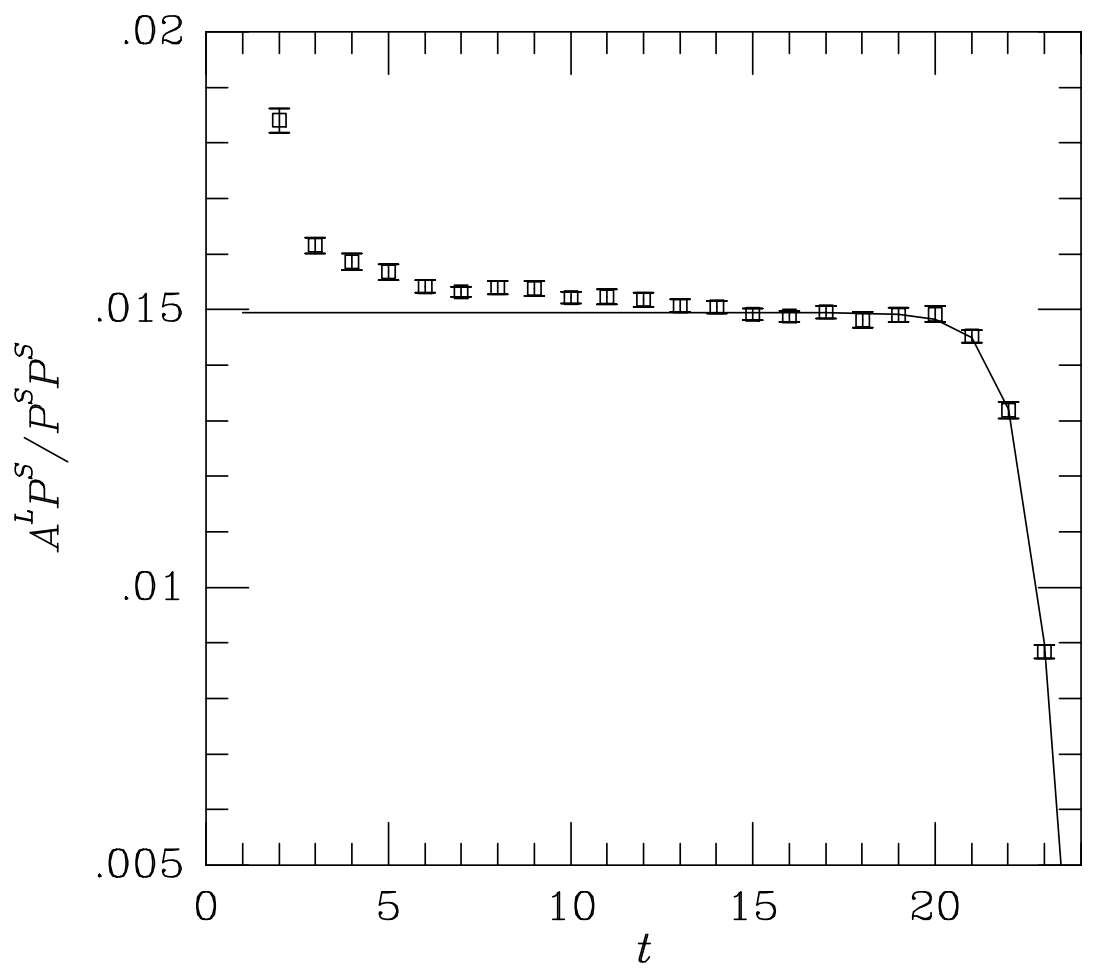

Figure 1: The ratio of correlators defined in eq. (20) plotted versus $t$ for $\kappa_{h}=0.129, \kappa_{l}=0.14262$. The curve represents the fit using timeslices $15-22$.

We start the discussion of our results with the behaviour of the pseudoscalar decay constants as a function of the mass of the meson, with all dimensionful quantities given in lattice units. In the heavy-quark limit, the quantity $f_{P} \sqrt{M_{P}}$ scales like

$$
f_{P} \sqrt{M_{P}}=\text { const. } \times\left[\alpha_{s}\left(M_{P}\right)\right]^{-2 / \beta_{0}}, \quad M_{P} \longrightarrow \infty .
$$

In order to detect possible deviations from this scaling law we plot in Fig. 2 the quantity

$$
\hat{\Phi}\left(M_{P}\right) \equiv\left(\alpha_{s}\left(M_{P}\right) / \alpha_{s}\left(M_{B}\right)\right)^{2 / \beta_{0}} Z_{A}^{-1} f_{P} \sqrt{M_{P}}
$$

\footnotetext{
${ }^{1}$ The normalization factor $\alpha_{s}\left(M_{B}\right)^{-2 / \beta_{0}}$ is convenient when comparing these results with those obtained in the static theory.
} 
as a function of $1 / M_{P}$. We approximate $\alpha_{s}(M)$ by

$$
\alpha_{s}(M)=\frac{2 \pi}{\beta_{0} \log \left(M / \Lambda_{\mathrm{QCD}}\right)}
$$

where we take $\Lambda_{\mathrm{QCD}}=200 \mathrm{MeV}$, and $\beta_{0}=11-\frac{2}{3} n_{f}$, with $n_{f}=0$ in the quenched approximation. From the figure we see that $\hat{\Phi}\left(M_{P}\right)$ increases as the mass of the heavy quark is increased (in agreement with the behaviour found using the Wilson action for the quarks [2, 3, 4). In order to quantify this behaviour, we fit $\hat{\Phi}\left(M_{P}\right)$ to either a linear or quadratic function of $1 / M_{P}$ :

$$
\hat{\Phi}\left(M_{P}\right)=A\left(1-\frac{B}{M_{P}}\right)
$$

or

$$
\hat{\Phi}\left(M_{P}\right)=C\left(1-\frac{D}{M_{P}}+\frac{E}{M_{P}^{2}}\right) .
$$

We have performed these fits twice; once using the values of $f_{P} \sqrt{M_{P}}$ for all four values of $\kappa_{h}$, and once using those for only the smallest three $\kappa_{h}$ 's (i.e. for the heaviest three heavy-quark masses). The results of the fits are given in Table 3. We find that the non-scaling corrections are of $O(30 \%)$ for $f_{D}$ and $O(10 \%)$ for $f_{B}$, in agreement with previous results obtained using Wilson fermions [2, 3, 4. From the quadratic fit to the data at all four heavy-meson masses we find, in physical units,

$$
\begin{gathered}
C a^{-3 / 2}=0.45^{+2}+19 \mathrm{GeV}^{3 / 2} \\
D a^{-1}=0.84 \begin{array}{l}
+11+22 \\
-8-3
\end{array} \mathrm{GeV} \\
E a^{-2}=0.28 \begin{array}{l}
+7+16 \\
-9-2
\end{array} \mathrm{GeV}^{2}
\end{gathered}
$$

The second error in eq. (26) corresponds solely to the uncertainty in the scale. It should be mentioned that ignoring the residual logarithmic dependence of $f_{P} \sqrt{M_{P}}$ on $M_{P}$ makes the slope more pronounced. However it is clear from Fig. 2 and Table 3 that the logarithmic corrections to the scaling law can by no means account for the observed slope in $f_{P} \sqrt{M_{P}}$.

We use the parameters of the fits in Table 3 to make our predictions for the values of the decay constants $f_{D}$ and $f_{B}$. The results corresponding to the four fits are presented in Table 1 . From this table it is clear that there is a further systematic uncertainty in $f_{B}$ of about $11 \mathrm{MeV}$ from extrapolating using either linear or quadratic fits. In contrast to this, since we interpolate to $m_{D}$, the results for $f_{D}$ are very stable. It should be emphasised that choosing a different value for $\Lambda_{\mathrm{QCD}}$ (e.g. $\Lambda_{\mathrm{QCD}}=250 \mathrm{MeV}$ ), or for the anomalous dimension (e.g., by taking $n_{f}=4$ ), changes the results by only about $1 \mathrm{MeV}$. 


\begin{tabular}{|c|c|c|c|c|c|}
\hline & \multicolumn{2}{|c|}{ Linear Fit } & \multicolumn{3}{|c|}{ Quadratic Fit } \\
\hline & $A$ & $B$ & $C$ & $D$ & $E$ \\
\hline $4 \kappa_{h}$ 's & $\begin{array}{l}0.089_{-2}^{+3} \\
-2\end{array}$ & $\begin{array}{l}0.199+5 \\
-7\end{array}$ & $\begin{array}{l}0.101_{-5}^{+5} \\
-5\end{array}$ & $\begin{array}{l}0.31_{-}^{+} 4 \\
-3\end{array}$ & $\begin{array}{l}0.038_{-13}^{+9} \\
-1\end{array}$ \\
\hline $3 \kappa_{h}$ 's & $0.092_{-3}^{+3}$ & $\begin{array}{r}0.216+9 \\
+ \\
\end{array}$ & $\begin{array}{r}0.102+6 \\
\end{array}$ & $0.33_{-}^{+10}$ & $\begin{array}{r}0.045+18 \\
-39 \\
\end{array}$ \\
\hline
\end{tabular}

Table 3: Values of the parameters of the linear and quadratic fits to the behaviour of the pseudoscalar decay constants with the mass of the mesons (as defined in the text).

\begin{tabular}{|c|c|c|c|c|}
\hline & \multicolumn{2}{|c|}{ Linear Fit } & \multicolumn{2}{|c|}{ Quadratic Fit } \\
\hline & $f_{D}$ & $f_{B}$ & $f_{D}$ & $f_{B}$ \\
\hline $4 \kappa_{h}$ 's & $185_{-3-4}^{+4+4}$ & $149 \begin{array}{l}+5+52 \\
-3-7\end{array}$ & $\begin{array}{l}185+4+42 \\
-3-7\end{array}$ & 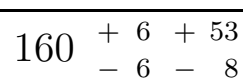 \\
\hline $3 \kappa_{h}$ 's & $186 \begin{array}{l}+4+4 \\
-3-3\end{array}$ & 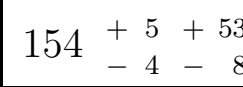 & $185 \begin{array}{l}+4+42 \\
-3-7\end{array}$ & $160 \begin{array}{l}+7+54 \\
-7-7\end{array}$ \\
\hline
\end{tabular}

Table 4: Values of the decay constants $f_{B}$ and $f_{D}$ in $\mathrm{MeV}$, corresponding to the linear and quadratic fits.

Taking the results from the quadratic fit using all four $\kappa_{h}$ values we find:

$$
\begin{array}{rl}
f_{D}=185 & +4+42 \\
& -3-7 \mathrm{MeV} \\
f_{B}=160 & +6+53 \\
-6-19 & \mathrm{MeV}
\end{array}
$$

where we have included the uncertainty of $11 \mathrm{MeV}$ from the extrapolations in the systematic error quoted for $f_{B}$. We take the results presented in equations (27) and (28) as our best estimates of the decay constants of the $D$ and $B$ mesons.

In ref. [3] it was found useful to use the pion decay constant, $f_{\pi}$, to set the scale in the computations of the decay constants of heavy-light mesons. By calculating $f_{D} / f_{\pi}$ and $f_{B} / f_{\pi}$ it may be expected that some of the systematic errors cancel, since, in particular, the ratios are independent of $Z_{A}$. Our results for the decay constants obtained in this way are, as expected, close to the upper systematic error margins in Table 4 . We find

$$
\begin{aligned}
& \left(\frac{f_{D}}{f_{\pi}}\right) \times 132 \mathrm{MeV}=232_{-}^{+12} \mathrm{MeV} \\
& \left(\frac{f_{B}}{f_{\pi}}\right) \times 132 \mathrm{MeV}=201 \stackrel{-12}{+} \mathrm{MeV} .
\end{aligned}
$$




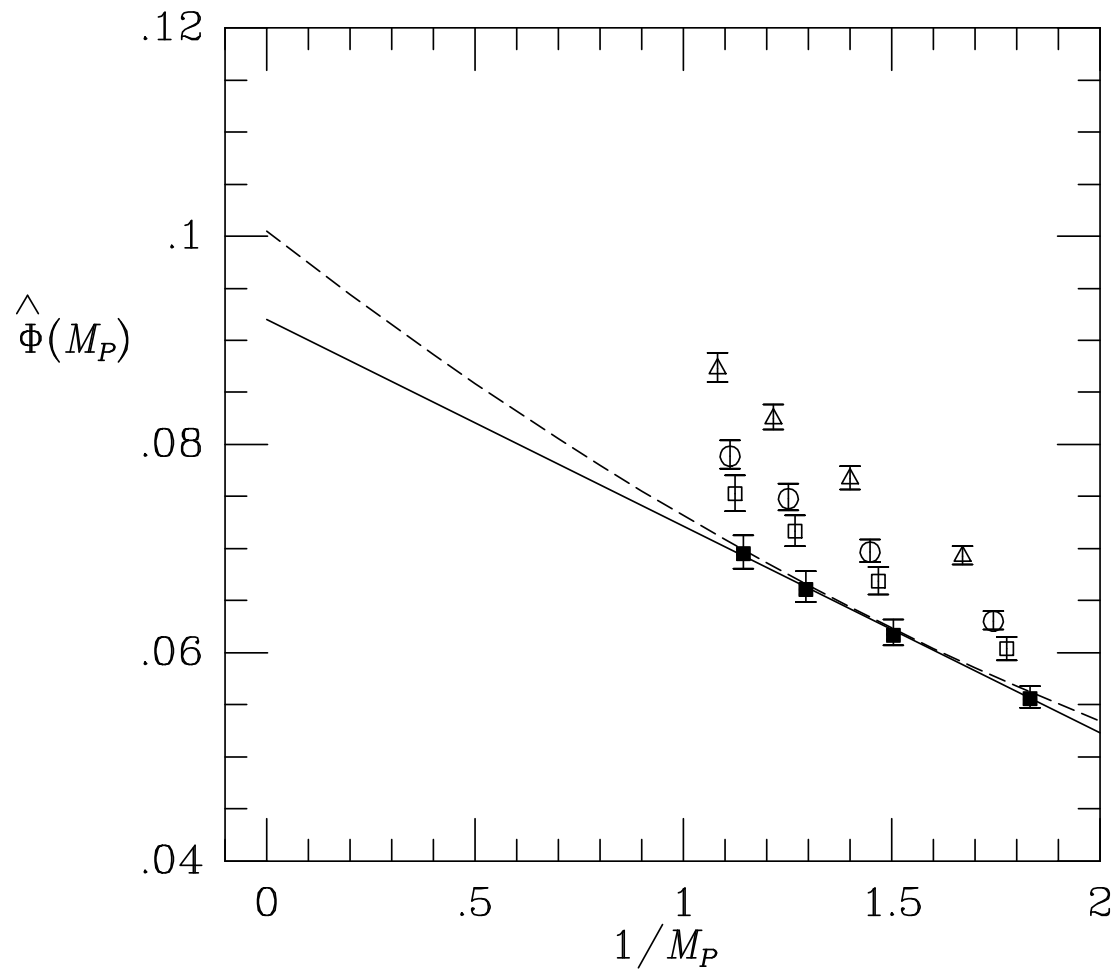

Figure 2: The data for $\hat{\Phi}\left(M_{P}\right)$ plotted against the inverse meson mass. The open symbols denote points with $\kappa_{l}<\kappa_{\text {crit }}$, whereas full symbols denote those extrapolated to $\kappa_{\text {crit }}$. The solid line represents the linear fit to the chirally-extrapolated points using the three heaviest meson masses, whereas the dashed curve results from a quadratic fit to all four.

Finally in this subsection, we present our results for $f_{D_{s}}$ and $f_{B_{s}}$. These are obtained by interpolating the measured values of the decay constants given in Table 2 to $\kappa_{l}=\kappa_{s}=$ $0.1419_{-1}^{+1}$ [15. The extrapolations in the heavy-quark masses are done as above. We find

$$
\begin{array}{r}
\frac{f_{D_{s}}}{f_{D}}=1.18_{-2}^{+2} \\
\frac{f_{B_{s}}}{f_{B}}=1.22_{-3}^{+4} .
\end{array}
$$


In physical units we obtain

$$
\begin{array}{r}
f_{D_{s}}=212 \begin{array}{l}
+4+46 \\
-4-7
\end{array} \mathrm{MeV} \\
f_{B_{s}}=194 \begin{array}{l}
+6+62 \\
-5-9
\end{array} \mathrm{MeV} .
\end{array}
$$

Recently the first measurement of $f_{D_{s}}$ has been made by the WA75 collaboration [27], who found $f_{D_{s}}=(232 \pm 45 \pm 20 \pm 48) \mathrm{MeV}$. Our result is in good agreement with the measured value, and also with previous lattice calculations using Wilson fermions [3, 5].

\subsection{Decay Constants of Vector Mesons}

In this subsection we present our results for the decay constants of heavy-light vector mesons. These are defined by

$$
\left\langle 0\left|V_{\mu}\right| V\right\rangle \equiv \epsilon_{\mu} \frac{M_{V}^{2}}{f_{V}}=Z_{V}\left\langle 0\left|V_{\mu}^{L}(0)\right| V\right\rangle
$$

where $|V\rangle$ represents a state containing a vector meson $V$, with mass $M_{V}$, polarisation vector

$\epsilon_{\mu}$ and decay constant $f_{V} . V_{\mu}^{L}$ denotes the local lattice vector current, defined in eq. (9), with $\Gamma=\gamma_{\mu}$, which has to be multiplied by the renormalisation constant $Z_{V}$. The vector mass $M_{V}$ is extracted from fits to the correlator

$$
\begin{aligned}
C_{V V}^{S S} & \equiv \sum_{j=1}^{3} \sum_{\vec{x}}\left\langle 0\left|V_{j}^{S}(\vec{x}, t) V_{j}^{S}(0)\right| 0\right\rangle \\
& \rightarrow-\frac{3 Z_{V^{S}}^{2}}{2 M_{V}} \exp \left(-M_{V} L_{t} / 2\right) \cosh \left(M_{V}\left(L_{t} / 2-t\right)\right)
\end{aligned}
$$

where $V_{j}^{S}$ is the $j$ th spatial component of the smeared vector operator and $Z_{V^{S}}$ is defined through

$$
\left\langle 0\left|V_{j}^{S}(0)\right| V\right\rangle=\epsilon_{j} Z_{V^{S}}
$$

Fitting timeslices $14 \leq t \leq 23$, symmetrized, we obtain the vector meson masses shown in Table 1. In order to extract the matrix element of the local vector current we fit the ratio

$$
\frac{C_{V V}^{L S}(t)}{C_{V V}^{S S}(t)} \longrightarrow-\frac{\sum_{j=1}^{3}\left\langle 0\left|V_{j}^{L}(0)\right| V\right\rangle \epsilon_{j}^{*}}{3 Z_{V^{S}}}
$$

to a constant in the fitting interval $15 \leq t \leq 23$.

The results for $1 / f_{V}$ for the twelve $\kappa_{h}-\kappa_{l}$ combinations are presented in Table 2, together with those obtained after extrapolation to the chiral limit. The chirally-extrapolated values 


\begin{tabular}{|c||c|c|}
\hline$M$ & Linear Fit & Quadratic Fit \\
\hline$\infty$ & $1.02_{-4}^{+5}$ & $1.09_{-8}^{+7}$ \\
\hline$\left(M_{B}+3 M_{B}^{*}\right) / 4$ & $0.93_{-3}^{+4}$ & $0.96_{-5}^{+4}$ \\
\hline$\left(M_{D}+3 M_{D}^{*}\right) / 4$ & $0.77_{-2}^{+2}$ & $0.77_{-2}^{+2}$ \\
\hline
\end{tabular}

Table 5: The quantity $\tilde{U}(M)$ obtained from linear and quadratic fits.

for $f_{V}^{-1} Z_{V}^{-1}$ are now interpolated to the $D^{*}$ mass using a quadratic fit to the data at all four values of $\kappa_{h}$, giving,

$$
f_{D^{*}}^{-1}=0.110 \begin{aligned}
& +5+36 \\
& -5-5
\end{aligned}
$$

This is slightly below, but still compatible with, earlier studies (e.g. [3]) when the systematic error is taken into account. This result remains unaltered if a linear fit is used instead of a quadratic one.

\subsection{A Test of the Heavy Quark Symmetry}

In the heavy-quark limit, the decay constants of heavy-light pseudoscalar and vector mesons are related by 28

$$
U(M) \equiv \frac{f_{V} f_{P}}{M}=\left(1+\frac{8}{3} \frac{\alpha_{s}(M)}{4 \pi}+O(1 / M)\right),
$$

where we take the heavy mass scale, $M$, to be the spin-averaged meson mass, $M=\left(M_{P}+\right.$ $\left.3 M_{V}\right) / 4$.

In order to test the predicted behaviour of $U(M)$, we take the chirally-extrapolated values for both the pseudoscalar and vector decay constants, and fit

$$
\tilde{U}(M) \equiv U(M) /\left\{1+\frac{8}{3} \frac{\alpha_{s}(M)}{4 \pi}\right\}
$$

to either a linear or quadratic function of $1 / M$. The data together with the fits are shown in Fig. 3, and we display our results in Table 5. The perturbative values of $Z_{A}$ and $Z_{V}$ are used.

The fact that $\tilde{U}(\infty)$ is around one in Table a provides support for our parametrisations, in eqs. (24) and (25), of the non-scaling behaviour of the decay constants for finite heavy-quark masses. 


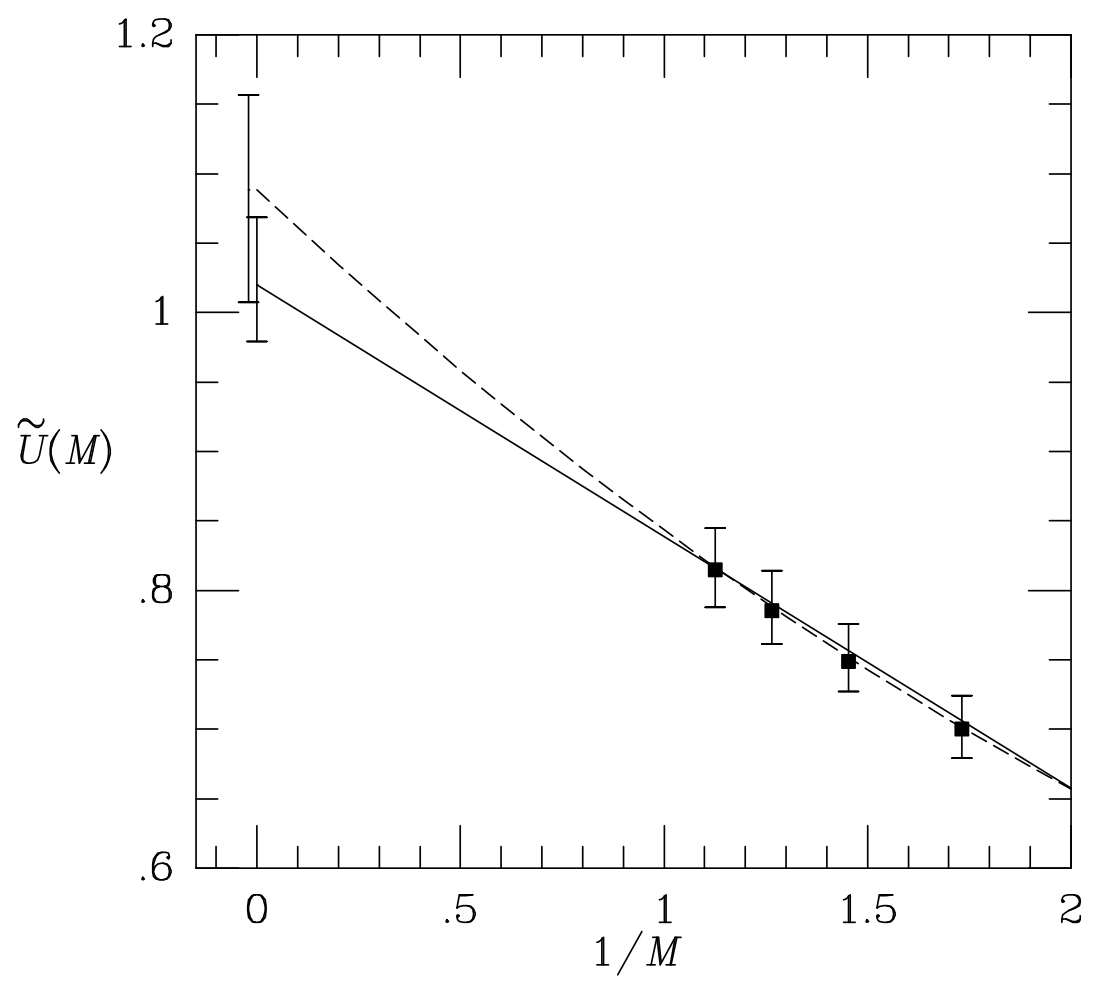

Figure 3: The quantity $\tilde{U}(M)$ plotted against the inverse spin-averaged mass. Linear and quadratic fits are represented by the solid and dashed curves, respectively. Also shown are the statistical errors of the extrapolation to the infinite mass limit.

\section{Decay Constants from the Simulation at $\beta=6.0$}

In this section we describe the results of a computation of the decay constants using the SW fermion action at $\beta=6.0$ on a $16^{3} \times 48$ lattice. These results were obtained using 36 configurations, with light-quark masses corresponding to $\kappa_{l}=0.1432,0.1440$ and 0.1445 . The corresponding light-light pseudoscalar and vector meson masses, and pseudoscalar decay constants, all in lattice units, are presented in Table 6. The values of the hopping parameter corresponding to the chiral limit and the strange quark mass are $\kappa_{\text {crit }}=0.14556{ }_{-6}^{+6}$ and 


\begin{tabular}{|c|c|c|c|}
\hline$\kappa_{l}$ & $m_{\pi}$ & $m_{\rho}$ & $f_{\pi} / Z_{A}$ \\
\hline 0.1432 & $0.386 \begin{array}{l}+4 \\
-4\end{array}$ & 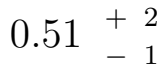 & 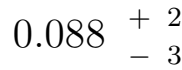 \\
\hline 0.1440 & $0.311+6$ & $0.47 \begin{array}{l}+3 \\
-2\end{array}$ & $0.080+2$ \\
\hline 0.1445 & $\begin{aligned} & 0.257+5 \\
&-6 \\
&\end{aligned}$ & $\begin{array}{r}0.43+6 \\
-3 \\
\end{array}$ & 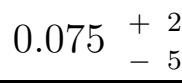 \\
\hline$\kappa_{\text {crit }}=0.14556 \begin{array}{l}+6 \\
-6\end{array}$ & - & $\begin{array}{l}0.38+5 \\
-4\end{array}$ & 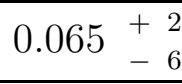 \\
\hline
\end{tabular}

Table 6: Masses of light-light pseudoscalar and vector mesons, and the pseudoscalar decay constants at $\beta=6.0$.

$\kappa_{s}=0.1437_{-5}^{+4}$ respectively. Using the mass of the $\rho$ meson to determine the value of the lattice spacing, we find $a^{-1}=2.0_{-2}^{+3} \mathrm{GeV}$, whilst using $f_{\pi}$ we find $a^{-1}=2.1_{-1}^{+2} \mathrm{GeV}$. These two results are compatible, and below we will use the value

$$
a^{-1}=2.0 \stackrel{+2}{+3} \mathrm{GeV}
$$

to convert the results from lattice to physical units.

We have computed the heavy-light correlation functions as series in $\kappa_{h}$ (the hopping-parameter expansion [29]), thus enabling us to obtain the decay constants at any value of the mass of the heavy quark, without explicitly computing the heavy-quark propagators. The decay constants are obtained by fitting to eq. (19) and eq. (20), over the range $12 \leq t \leq 18$ for both fits. We employ the Jacobi smearing algorithm with $N=50$, corresponding to a smearing radius of $r=4.2$.

In an attempt to improve our understanding of the discretisation errors, we have also computed the decay constants for the Wilson action at one value of the light-quark mass, using a subset of 16 of the 36 configurations. The comparison of the results for the two actions is presented in Subsection 3.2 .

\subsection{Pseudoscalar Decay Constants}

In Fig. 1 we plot the chirally-extrapolated values of $\hat{\Phi}\left(M_{P}\right)$ as a function of $1 / M_{P}$, for 11 values of the heavy-quark mass. We fit the points corresponding to the five lightest meson masses (for which $m_{Q}=1 / 2\left(1 / \kappa_{h}-1 / \kappa_{\text {crit }}\right)<0.7$, as was the case at $\beta=6.2$ ) to eq. (25), 
and this is shown as the solid curve in the figure. For the coefficients of the fit we find:

$$
C=0.18_{-3}^{+3} ; \quad D=0.45_{-5}^{+13} ; \quad E=0.08 \stackrel{+9}{+9}
$$

In physical units we obtain

$$
\begin{aligned}
& C a^{-3 / 2}=0.50 \begin{array}{l}
+9+12 \\
-9
\end{array} \mathrm{GeV}^{3 / 2} \\
& D a^{-1}=0.91+21+14 \\
&-26-9 \\
& \mathrm{GeV} \\
& E a^{-2}=0.32 \begin{array}{l}
+16+10 \\
-36-6
\end{array} \mathrm{GeV}^{2}
\end{aligned}
$$

in good agreement with the results at $\beta=6.2$, quoted in eq. (26). It should be noted that the inclusion of all 11 data points makes no significant difference to the fit.

The values for the decay constants in physical units are:

$$
\begin{aligned}
& f_{D}=199 \begin{array}{l}
+14+27 \\
-15-19
\end{array} \\
& \mathrm{MeV} \\
& f_{B}=176 \begin{array}{l}
+25+33 \\
-24-15
\end{array} \mathrm{MeV} \\
& \frac{f_{D_{s}}}{f_{D}}=1.13_{-7}^{+6} \\
&-7 \\
& \frac{f_{B_{s}}}{f_{B}}=1.17_{-12}^{+12} \\
&-12
\end{aligned}
$$

All these numbers are in good agreement with the corresponding results from the simulation at $\beta=6.2$. Finally, for the ratios $f_{D} / f_{\pi}$ and $f_{B} / f_{\pi}$ we obtain

$$
\begin{aligned}
& \left(\frac{f_{D}}{f_{\pi}}\right) \times 132 \mathrm{MeV}=211_{-11}^{+26} \mathrm{MeV} \\
& \left(\frac{f_{B}}{f_{\pi}}\right) \times 132 \mathrm{MeV}=186_{-21}^{+35} \mathrm{MeV} .
\end{aligned}
$$

\subsection{Comparison of Results Using Wilson and SW Actions}

For 16 of the configurations, we have computed the decay constants for the Wilson fermion action, again using the hopping-parameter expansion. We compute light-quark propagators at a single value of the hopping parameter, $\kappa_{l}^{W}=0.155$, corresponding to a pseudoscalarmeson mass of $0.30+1$. This was chosen to match the SW pseudoscalar-meson mass of $0.31+2$ obtained at $\kappa_{l}^{\mathrm{SW}}=0.144$ on the same set of configurations. 


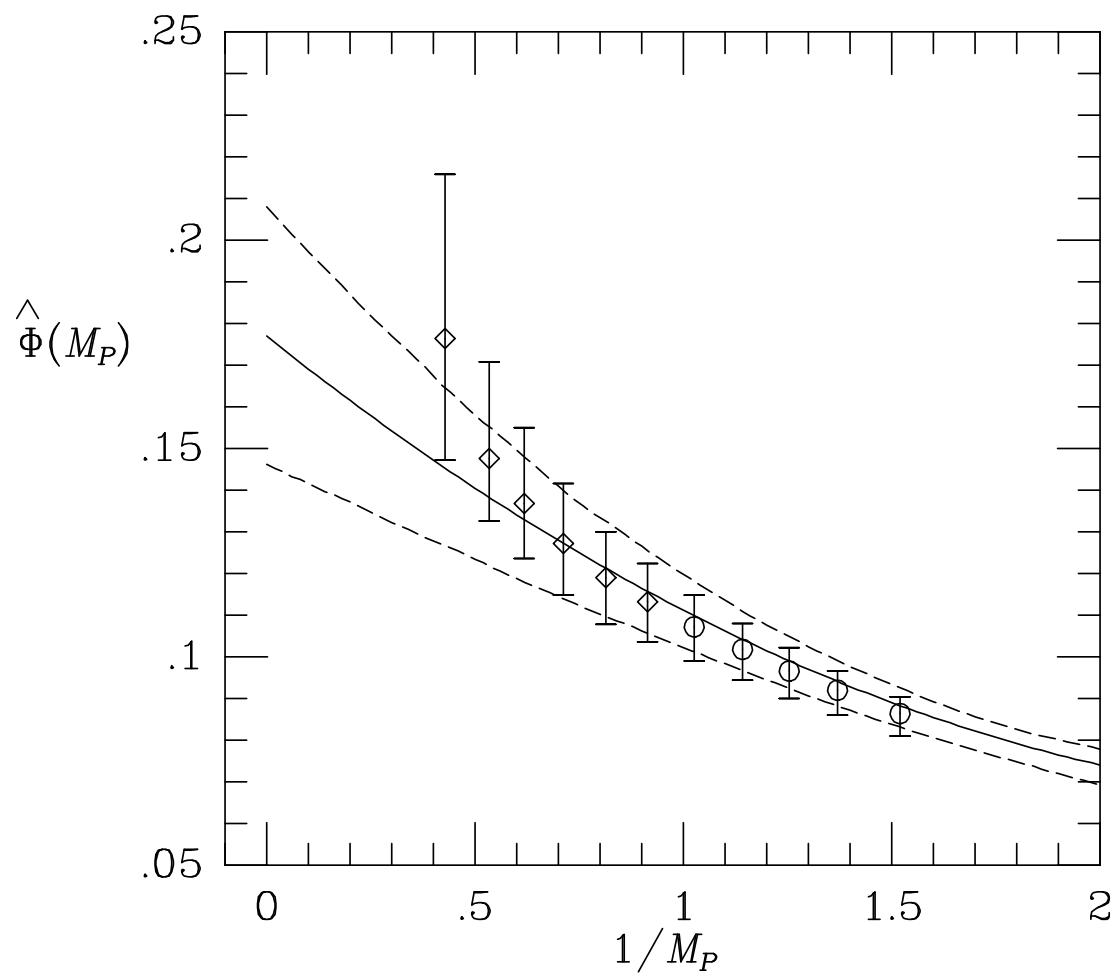

Figure 4: The chirally-extrapolated data for $\hat{\Phi}\left(M_{P}\right)$ at $\beta=6.0$ plotted against the inverse meson mass. The solid curve represents a quadratic fit to the points denoted by circles. Points represented by diamonds are not included in the fit. The dashed curves are the $68 \%$ confidence bounds on the fit.

In Fig. 5 we plot $f_{P} \sqrt{M_{P}}$ as a function of $1 / M_{P}$. Using the conventional normalisation of $\sqrt{2 \kappa}$ for the quark fields, we see a clear divergence between the results for the two actions for $m_{Q}>0.7$; the Wilson results turn over and decrease. However, we note that uncorrelated $\chi^{2}$ fits of the Wilson points, at the lightest few meson masses, to eqs. (24) and (25) would yield coefficients of the $1 / M_{P}$ term broadly consistent with previous Wilson analyses.

The figure also shows the results obtained with the Wilson action, but using the normalisation $\sqrt{1-6 \tilde{\kappa}}$ for the quark fields [5, 19, 30], where $\tilde{\kappa}=u_{0} \kappa$ and $u_{0}=1 /\left(8 \kappa_{\text {crit }}\right)$. It has been suggested that this normalisation may absorb some of the discretisation errors [19], 


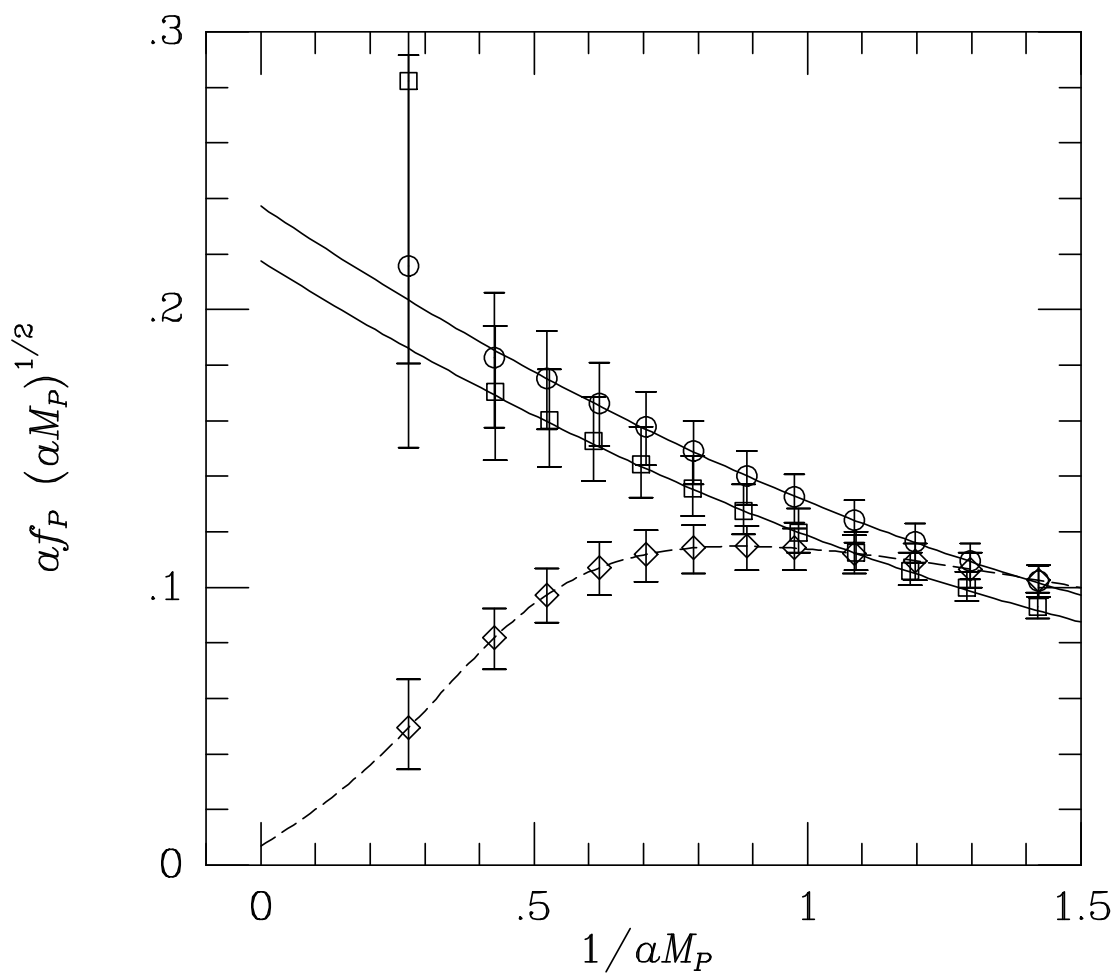

Figure 5: $f_{P} \sqrt{M_{P}}$ for both the Wilson and SW actions. Diamonds denote points obtained with the Wilson action in the conventional normalisation, $\sqrt{2 \kappa}$, whereas squares denote points normalised by $\sqrt{1-6 \tilde{\kappa}}$. Results using the SW action are represented by circles. The solid curves are quadratic fits in $1 / M_{P}$ to $f_{P} \sqrt{M_{P}}$ for the Wilson action, with fields normalized by $\sqrt{1-6 \tilde{\kappa}}$, and for the SW action. The dashed curve is to guide the eye.

and indeed the corresponding results agree remarkably with those obtained using the SW action. This agreement provides considerable motivation for a theoretical study to investigate whether there is any formal connection between the ansatz above and the improvement programme initiated by Symanzik [31. 


\section{$4 f_{B}$ in the Static Limit}

An alternative and complementary approach to heavy-quark physics using lattice QCD was proposed by Eichten [16]. This technique is based on an expansion of the heavy-quark propagator in inverse powers of the quark mass. In practice, one keeps just the leading term, given by (at zero velocity)

$$
S_{Q}(\vec{x}, t ; \overrightarrow{0}, 0)=\left\{\theta(t) e^{-m_{Q} t} \frac{1+\gamma^{4}}{2}+\theta(-t) e^{m_{Q} t} \frac{1-\gamma^{4}}{2}\right\} \delta^{(3)}(\vec{x}) \mathcal{P}_{\overrightarrow{0}}(t, 0),
$$

where $\mathcal{P}_{\overrightarrow{0}}(t, 0)$ is the product of links from $(\overrightarrow{0}, t)$ to the origin, for example for $t>0$,

$$
\mathcal{P}_{\overrightarrow{0}}(t, 0)=U_{4}^{\dagger}(\overrightarrow{0}, t-1) U_{4}^{\dagger}(\overrightarrow{0}, t-2) \cdots U_{4}^{\dagger}(\overrightarrow{0}, 0) .
$$

At sufficiently large times

$$
\sum_{\vec{x}}\left\langle A_{4}(\vec{x}, t) A_{4}^{\dagger}(0)\right\rangle \rightarrow \frac{f_{P}^{2} M_{P}}{2} e^{-M_{P} t},
$$

where $A_{\mu}$ is the improved axial current of eq. (10) with $\Gamma=\gamma_{\mu} \gamma_{5}$. Since the only dependence on $m_{Q}$ in eq. (53) arises through the exponential factor in eq. (51), we deduce the scaling law that $f_{P} \sqrt{M_{P}}$ is independent of the heavy-quark mass. Matching the result from the Heavy Quark Effective Theory with that in the full theory introduces the logarithmic corrections in eq. (21). The full scaling law is of the form

$$
f_{P} \sqrt{M_{P}}=\text { const. }\left[\left(\alpha_{s}\left(M_{P}\right)\right)^{-2 / \beta_{0}}\left(1+O\left(\alpha_{s}\right)\right)+O\left(1 / M_{P}\right)\right] .
$$

The objective of lattice computations is to determine the constant. We refer to the value of $f_{B}$ obtained using eq. (54), but dropping the $O\left(1 / M_{B}\right)$ corrections, as $f_{B}^{\text {stat }}$.

We compute the two correlation functions, $C^{S S}$ and $C^{L S}$, defined by ${ }^{2}$

$$
\begin{aligned}
C^{S S}(t) & =\sum_{\vec{x}}\left\langle 0\left|A_{4}^{S}(\vec{x}, t) A_{4}^{\dagger S}(\overrightarrow{0}, 0)\right| 0\right\rangle \rightarrow\left(Z^{S}\right)^{2} e^{-\Delta E t} \\
C^{L S}(t) & =\sum_{\vec{x}}\left\langle 0\left|A_{4}^{L}(\vec{x}, t) A_{4}^{\dagger S}(\overrightarrow{0}, 0)\right| 0\right\rangle \rightarrow Z^{L} Z^{S} e^{-\Delta E t}
\end{aligned}
$$

where $\Delta E$ is the (unphysical) difference between the mass of the meson and the bare mass of the heavy quark. The matrix element of the local operator $A_{4}^{L}$ is obtained from the two correlation functions $C^{S S}$ and $C^{L S}$ as follows. By fitting $C^{S S}(t)$ to the functional form given in eq. (55) we obtain $Z_{S}$ (and $\Delta E$ ). At sufficiently large times the ratio $C^{L S}(t) / C^{S S}(t) \rightarrow$ $Z^{L} / Z^{S}$, so that $Z^{L}$ can be determined.

\footnotetext{
${ }^{2}$ In principle the behaviour of the correlation functions in eqs. (55) and (56) is given by a cosh (as in eq. (19)), however the contribution of the backward-propagating meson is negligible in the time intervals we will be considering.
} 


\begin{tabular}{|c|c|c|c|c|c|c|c|}
\hline$\kappa_{l}$ & $\Delta E$ & \multicolumn{2}{|c|}{$\left(Z^{S}\right)^{2}$} & \multicolumn{2}{|c|}{$Z^{L} / Z^{S}$} & \multicolumn{2}{|c|}{$Z^{L}$} \\
\hline & $N=140$ & $N=110$ & $N=140$ & $N=110$ & $N=140$ & $N=110$ & $N=140$ \\
\hline 0.14144 & $\begin{array}{l}+1 \\
0.59\end{array}$ & $\begin{array}{l}141_{-11}^{+12} \\
-12\end{array}$ & $130 \begin{array}{l}+10 \\
-11\end{array}$ & $\begin{array}{l}0.0125_{-3}^{+3} \\
\end{array}$ & $0.0039_{-1}^{+1}$ & $0.149_{-7}^{+7}$ & $0.142_{-6}^{+7}$ \\
\hline 0.14226 & $0.57+1$ & $127+12$ & $119_{-11}^{+10}$ & $0.0121+3$ & $0.0038^{+1}$ & $0.137^{+6}$ & $0.130+7$ \\
\hline 0.14262 & $0.56_{-1}^{+2}$ & $119_{-11}^{+12}$ & $112_{-11}^{+9}$ & $0.0120_{-3}^{+3}$ & $0.0037_{-1}^{+1}$ & $0.131_{-6}^{+7}$ & $0.125_{-7}^{+7}$ \\
\hline
\end{tabular}

Table 7: Values of $\Delta E,\left(Z^{S}\right)^{2}, Z^{L} / Z^{S}$ and $Z^{L}$ at the three value of $\kappa_{l} . \Delta E$ is obtained from the fit to $C^{S S}(t)$.

\subsection{Results at $\beta=6.2$}

We now report on a computation of $f_{B}^{\text {stat }}$ at $\beta=6.2$. The results presented here were obtained using a subset of 20 of the 60 configurations discussed in Section 2, at the three values of the light-quark mass. The values of $f_{B}^{\text {stat }}$ and $f_{B_{s}}^{\text {stat }}$ were determined by extrapolating the results to $\kappa_{\text {crit }}$ and $\kappa_{s}$ respectively.

In view of the difficulty in isolating the ground state in correlation functions using the static effective theory, we have compared results obtained with different numbers of iterations of the Jacobi smearing algorithm [33. For $N$ less than about 80 the plateaus do not start until at least $t=7$. In this paper we present our results obtained with $N=110$ and $N=140$, corresponding to $r=5.9$ and 6.4 respectively, where plateaus begin as early as $t=4$ and hence statistical errors are smaller.

In Fig. 6(a) we show the effective masses obtained from $C^{S S}(t)$, and in Fig. 6(b) the ratio $C^{L S}(t) / C^{S S}(t)$, both at $\kappa_{l}=0.14226$ and $N=140$. Excellent plateaus are obtained, giving us confidence that the ground state has indeed been isolated. In Table 0 we present the results for $\Delta E,\left(Z^{S}\right)^{2}, Z^{L} / Z^{S}$ and $Z^{L}$ at all three values of $\kappa_{l}$, from fits over the range $5 \leq t \leq 11$, without symmetrization in Euclidean time. $\Delta E$ is obtained from the fit to $C^{S S}(t)$ for $N=140$; consistent values are obtained for $N=110$.

Extrapolating the results for $Z^{L}$ in Table 7 to the chiral limit and to the mass of the strange quark we find:

$$
\begin{aligned}
& Z^{L}=0.124_{-7}^{+8} \text { at } \kappa_{l}=\kappa_{\text {crit }} \\
& Z^{L}=0.140_{-6}^{+7} \text { at } \kappa_{l}=\kappa_{s}
\end{aligned}
$$



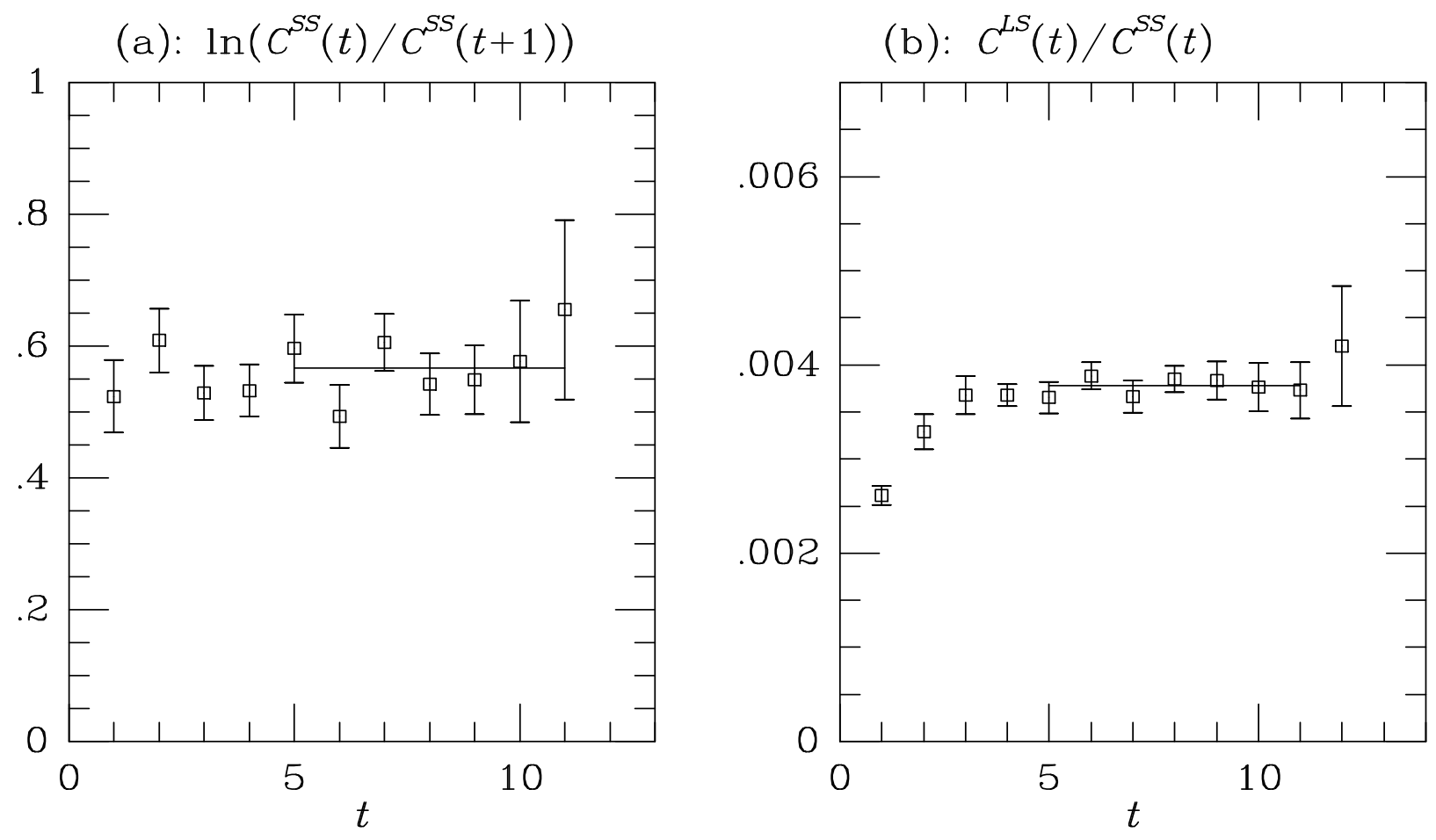

Figure 6: (a) The effective mass obtained from $C^{S S}(t)$, and (b) the ratio $C^{L S}(t) / C^{S S}(t)$ at $\beta=6.2, \kappa_{l}=0.14226$ and $N=140$. The solid lines represent fits from $5 \leq t \leq 11$.

when obtained using smeared interpolating operators with $N=110$ and

$$
\begin{aligned}
Z^{L}=0.117 \begin{array}{l}
+7 \\
-7
\end{array} \text { at } \kappa_{l}=\kappa_{\text {crit }} \\
Z^{L}=0.134 \begin{array}{l}
+7 \\
-6
\end{array} \text { at } \kappa_{l}=\kappa_{s}
\end{aligned} \text { a }
$$

when using interpolating operators with $N=140$.

When matching the static lattice theory to the full theory at a scale $m_{b}$, the factor required is [35]:

$$
Z_{A}^{\text {stat }}=\mathcal{Z}_{A}\left(1+\frac{\alpha_{s}\left(a^{-1}\right)}{3 \pi}\left[\frac{3}{2} \log a^{2} m_{b}^{2}-2\right]\right)
$$


$\mathcal{Z}_{A}$, relating the axial current in the static lattice theory to the static continuum one, has been calculated in perturbation theory for the SW action [34, 17]:

$$
\mathcal{Z}_{A}=1-0.127 g^{2} \simeq 0.79 .
$$

The value of 0.79 on the right hand side of eq. (62) was estimated using the boosted coupling at $\beta=6.2$. For the remaining factor in eq. (61), we take $m_{b}=5 \mathrm{GeV}, \alpha_{s}$ given by eq. (23) with $n_{f}=0$, and $\Lambda_{\mathrm{QCD}}=200 \mathrm{MeV}$, yielding a number close to one (note that this is insensitive to small changes in $m_{b}$ ). Thus $Z_{A}^{\text {stat }}=0.79$ also. We find

$$
\begin{aligned}
& f_{B}^{\text {stat }}=266 \begin{array}{l}
+17+110 \\
-15-14
\end{array}\left(\frac{Z_{A}^{\text {stat }}}{0.79}\right) \mathrm{MeV} \\
& f_{B_{\mathrm{s}}}^{\text {stat }}=300 \begin{array}{l}
+14+125 \\
-13-16
\end{array}\left(\frac{Z_{A}^{\text {stat }}}{0.79}\right) \mathrm{MeV},
\end{aligned}
$$

when using the interpolating operators with $N=110$, and

$$
\begin{aligned}
& f_{B}^{\text {stat }}=253 \begin{array}{l}
+16+105 \\
-15-14
\end{array}\left(\frac{Z_{A}^{\text {stat }}}{0.79}\right) \mathrm{MeV} \\
& f_{B_{\mathrm{s}}}^{\text {stat }}=287 \begin{array}{l}
+14+119 \\
-13-15
\end{array}\left(\frac{Z_{A}^{\text {stat }}}{0.79}\right) \mathrm{MeV}
\end{aligned}
$$

when using those with $N=140$. The systematic errors quoted arise from the uncertainty in the scale. We take the results in equations (65) and (66) as our best values, and these give for the ratio:

$$
\frac{f_{B_{\mathrm{s}}}^{\mathrm{stat}}}{f_{B}^{\mathrm{stat}}}=1.14_{-3}^{+4}
$$

\subsection{Results at $\beta=6.0$}

We have performed a similar analysis on the 36 configurations at $\beta=6.0$, using Jacobi smearing with $N=50$ and 150 , corresponding to $r=4.2$ and 6.2 respectively. The results obtained using the two smearing radii are consistent, and our best results are those at $N=50$ for which $Z_{L}=0.211_{-7}^{+6}$, yielding

$$
\begin{aligned}
f_{B}^{\text {stat }} & =286 \begin{array}{l}
+8+67 \\
-10-42
\end{array}\left(\frac{Z_{A}^{\text {stat }}}{0.78}\right) \mathrm{MeV} \\
f_{B_{\mathrm{s}}}^{\text {stat }} & =323{ }_{-14}^{+14+75}\left(\frac{Z_{A}^{\text {stat }}}{0.78}\right) \mathrm{MeV} \\
\frac{f_{B_{\mathrm{s}}}^{\text {stat }}}{f_{B}^{\text {stat }}} & =1.13_{-3}^{+4}
\end{aligned}
$$



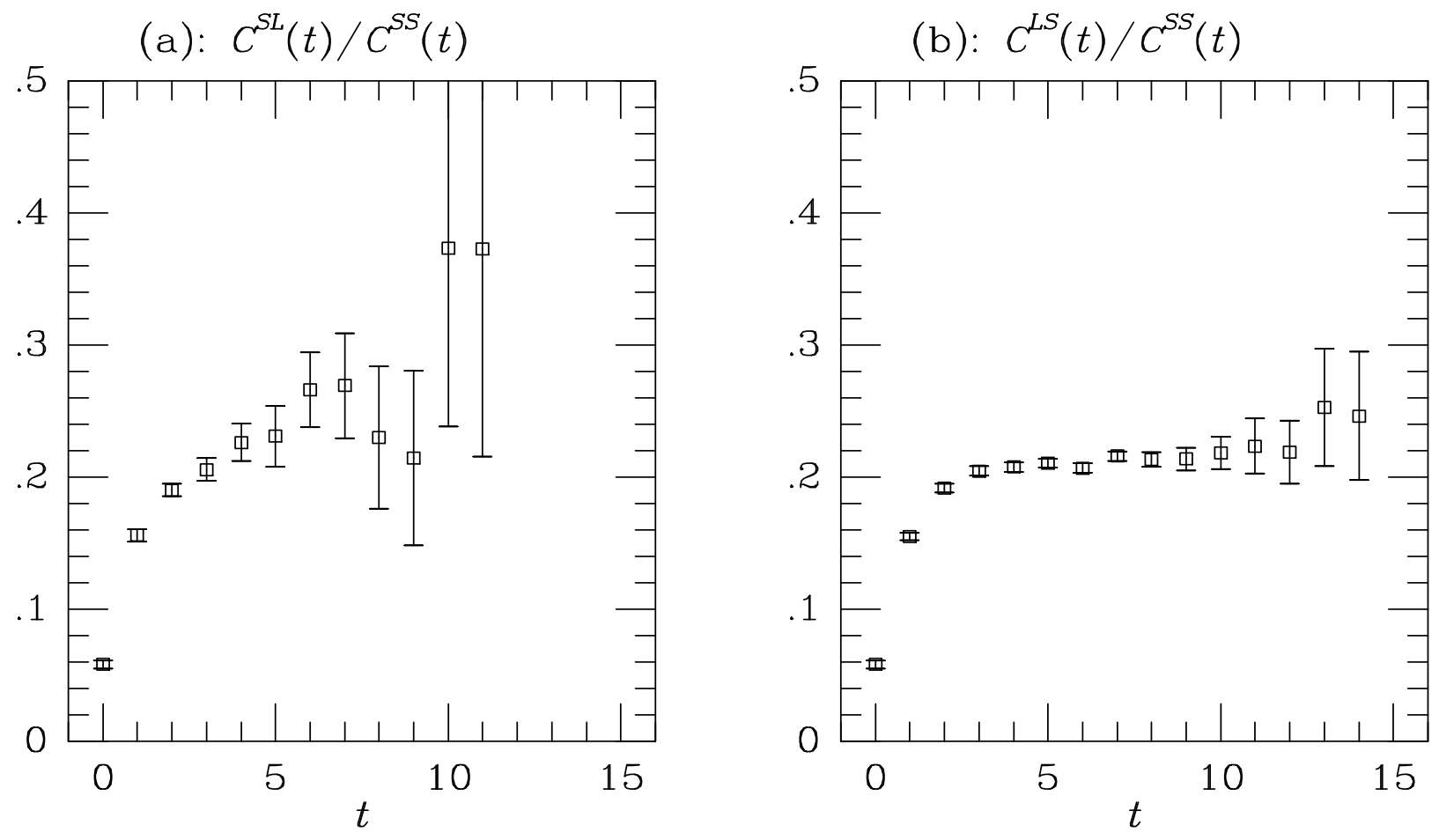

Figure 7: (a) The ratio $C^{S L}(t) / C^{S S}(t)$ and (b) $C^{L S}(t) / C^{S S}(t)$ plotted against $t$ for $\beta=6.0, \kappa_{l}=0.1432$, and $N=50$ iterations used in the Jacobi smearing algorithm.

These results at $\beta=6.0$ are consistent with those at $\beta=6.2$ presented in eqs. (65), (66) and (67).

The results plotted in Fig. 6(b) for the ratio $C^{L S}(t) / C^{S S}(t)$ appear to have considerably smaller errors, and a clearer plateau, than in some recent studies [8, 10], in spite of our limited statistics. We attribute this to the fact that we use the $C^{L S}$ correlation function in which the smearing is performed at the source, rather than the $C^{S L}$ correlation function in which the smearing is performed at the sink 3 . Of course, with sufficiently many configurations, the results are independent of this choice. However in the $C^{L S}$ correlation function, the

\footnotetext{
${ }^{3}$ In both refs. [8] and [10] it was in fact the $C^{S L}$, and not the $C^{L S}$, correlation function which was computed [36].
} 
heavy-quark propagator is sampled at many spatial points, whereas in the $C^{S L}$ correlation function only the heavy-quark propagator at $\vec{x}=\overrightarrow{0}$ contributes. Thus it seems plausible that the statistical errors are considerably reduced using the $C^{L S}$ correlation function.

To check this hypothesis, we have computed the ratios $C^{L S}(t) / C^{S S}(t)$ and $C^{S L}(t) / C^{S S}(t)$ at $\beta=6.0$, with $\kappa_{l}=0.1432$. The results are plotted in Fig. 0, and indeed confirm that there is an enormous improvement in precision when the correlation function $C^{L S}$ is used. We believe that this, rather than the different method of smearing, is the reason for the relatively poor plateaus in ref. [10].

\subsection{Discussion}

We begin with a comparison of the static and propagating results. Because we do not yet have static results for the full set of configurations at $\beta=6.2$, we focus on a comparison at $\beta=6.0$. In Fig. 8 we plot our results for the scaling quantity $f_{P} \sqrt{M_{P}}\left(\alpha\left(M_{P}\right) / \alpha\left(M_{B}\right)\right)^{6 / 33}$ from the simulation at $\beta=6.0$ as a function of $1 / M_{P}$ (in lattice units), together with our result for $f_{B}^{\text {stat }} \sqrt{M_{B}}$. The quadratic fit which we used to obtain our estimate for $f_{B}$ in Subsection 3.1 gives an intercept at $1 / M_{P}=0$ which is about $25 \%$ and two standard deviations below the static result; a similar discrepancy is observed at $\beta=6.2$. There are a number of possible reasons for this, e.g. uncertainties in the renormalisation constants (which are different for the static and propagating quarks), residual discretisation errors in the simulation of the propagating quarks, and uncertainties in the various extrapolations.

A better way of determining the consistency of the static and propagating results is to include the static result in the quadratic fit. Such a fit using the full correlation matrix at $\beta=6.0$ gives a $\chi^{2} /$ dof of 1.5 . This is still acceptable, and provides further evidence that using rotated operators with the SW action gives a sensible normalisation for propagating

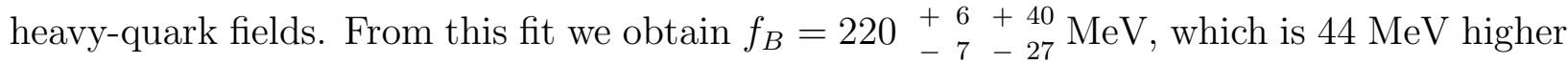
than that obtained from the propagating points alone.

In Table 8 we present the results for $f_{B}^{\text {stat }}$ obtained by other groups, together with our values. Although at $\beta=6.0$ the values of $Z^{L}$ found by all groups and for both actions are in broad agreement, the different treatment of systematics leads to the spread of results in $f_{B}^{\text {stat }}$. It has been suggested that $f_{B}^{\text {stat }}$ decreases as $a \rightarrow 0$ [12]. However, the agreement of the results obtained with the Wilson and SW actions at $\beta=6.0$, together with consistency between our results at $\beta=6.0$ and $\beta=6.2$, suggests that the discretisation errors are small. 


\begin{tabular}{|c|c|c|c|c|c|}
\hline Ref. & Action & $\beta$ & $a^{-1}[\mathrm{GeV}]$ & $Z_{A}^{\text {stat }}$ & $f_{B}^{\text {stat }}[\mathrm{MeV}]$ \\
\hline 111 & Wilson & 5.9 & 1.75 & 0.79 & $319 \pm 11$ \\
\hline 8 & Wilson & 6.0 & $2.0 \pm 0.2$ & 0.8 & $310 \pm 25 \pm 50$ \\
\hline 9 & Wilson & 6.0 & $2.2 \pm 0.2$ & 0.8 & $366 \pm 22 \pm 55$ \\
\hline 10 & Wilson & 6.0 & $2.11 \pm 0.05 \pm 0.10$ & 0.8 & $350 \pm 40 \pm 30$ \\
\hline 10 & SW & 6.0 & $2.05 \pm 0.06$ & 0.89 & $370 \pm 40$ \\
\hline This Work & SW & 6.0 & $2.0_{-2}^{+3}$ & 0.78 & $286 \begin{array}{r}+8+67 \\
-10-42\end{array}$ \\
\hline This Work & SW & 6.2 & 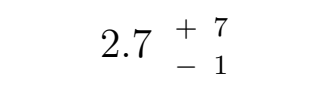 & 0.79 & 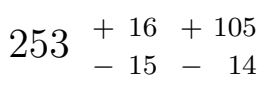 \\
\hline 5 & Wilson & 6.3 & $3.21 \pm .09 \pm .17$ & 0.69 & $235 \pm 20 \pm 21$ \\
\hline $\mid 12$ & Wilson & $5.74,6.0,6.26$ & $1.12,1.88,2.78$ & $0.71(8)$ & $230 \pm 22 \pm 26$ \\
\hline
\end{tabular}

Table 8: Compilation of lattice results for $f_{B}^{\text {stat }}$

\section{Conclusions}

In this paper we have carried out an extensive study of the decay constants of heavy-light mesons using the SW action for the quarks. The use of the SW action confirms the large, negative $O\left(1 / M_{P}\right)$ corrections to the scaling law $f_{P} \sqrt{M_{P}} \sim$ constant at the mass of the charm quark and the significant corrections at the mass of the $b$ quark, previously observed with the Wilson action. However, from our comparison of results for the Wilson and SW actions at $\beta=6.0$, we observe clear evidence that the $\sqrt{2 \kappa}$ normalisation of the Wilson quark fields fails for large quark mass. This failure is presumably due to large $O\left(m_{Q} a\right)$ effects. It has been suggested that such effects may largely be absorbed by the use of a different normalization [5]. We find that such a normalization yields results in agreement with those obtained using the SW action with rotated operators and the $\sqrt{2 \kappa}$ normalization for the quark fields.

Our best estimates of $f_{D}$ and $f_{B}$ are

$$
\begin{aligned}
& f_{D}=185 \begin{array}{l}
+4 \\
-3
\end{array} \text { (stat) } \begin{array}{l}
+42 \\
-7 \text { (syst }) \mathrm{MeV}
\end{array} \\
& f_{B}=160 \begin{array}{l}
+6 \\
-6
\end{array}-53 \mathrm{MeV} \\
&-6
\end{aligned}
$$

obtained using propagating quarks at $\beta=6.2$. Our analysis at $\beta=6.0$ yields entirely consistent results. The latter analysis also suggests that including the static result in the fits is likely to increase the value of $f_{B}$ by around $40 \mathrm{MeV}$. 


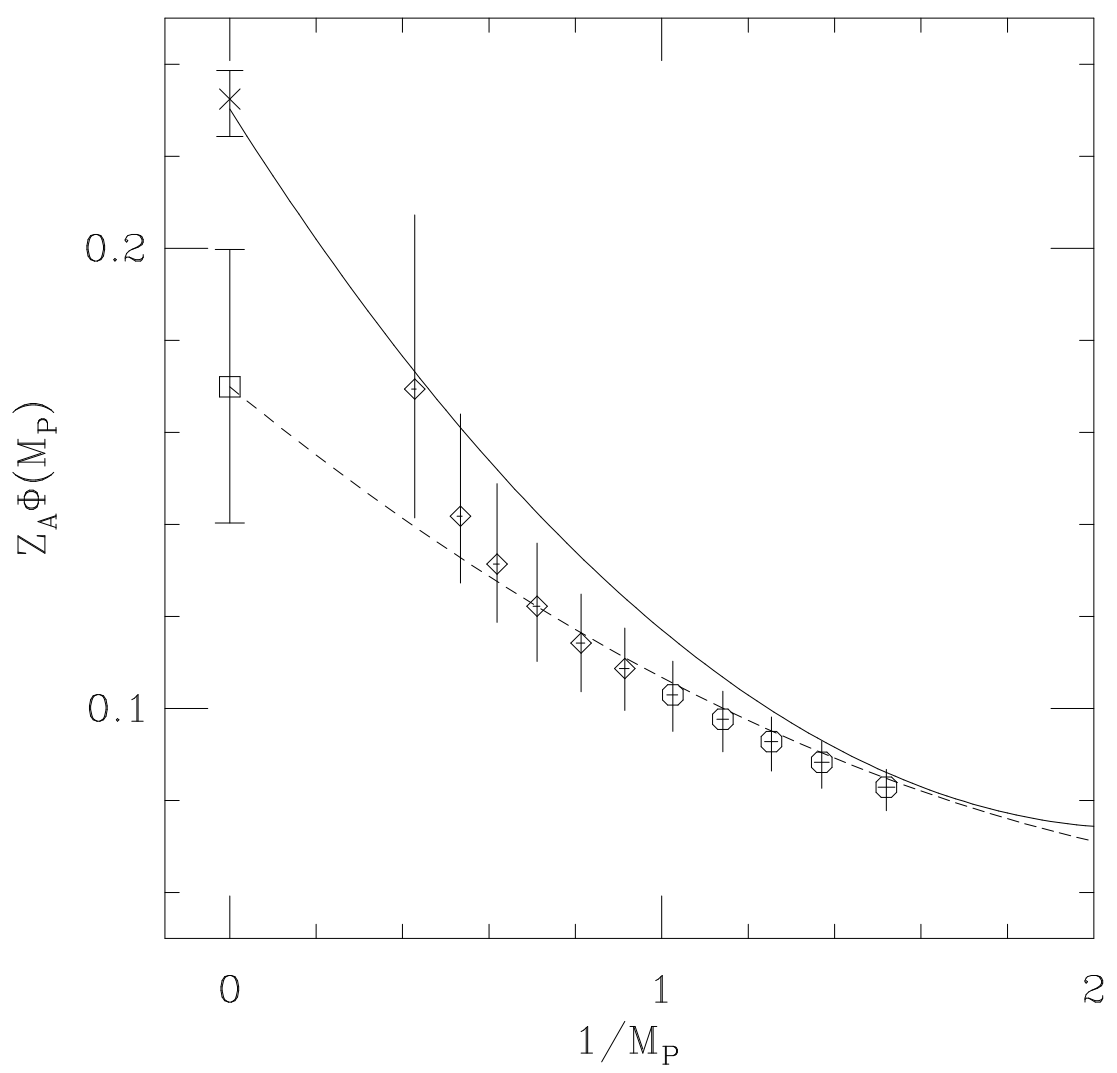

Figure 8: $Z_{A} \hat{\Phi}\left(M_{P}\right)$ at $\beta=6.0$ from the simulation with propagating quarks (open symbols) and the static theory (cross). The dashed curve is the fit to the open circles, with the parameters of eq. (43); the square is the intercept at $1 / M_{P}=0$. The solid curve is the fit with the static point included.

The most urgent extension of this work is to determine the $B$ parameter for $B^{0}-\bar{B}^{0}$ mixing, since it is the combination $f_{B} \sqrt{B_{B}}$ which is directly relevant for phenomenological studies of the mixing and of $C P$-violation. A recent simulation with Wilson fermions found $B_{B}=$ $1.16 \pm 0.07$ [3], and it is important to confirm this result with the improved action.

Acknowledgements We are grateful to G. Martinelli for many helpful discussions. CTS (Senior Fellow) and ADS (Personal Fellow) acknowledge the support of the Science and Engineering Research Council. This work was supported by SERC Grants GR/32779, GR/H 49191, GR/H 01069, GR/H 53624, the University of Edinburgh and Meiko Limited. We are 
grateful to Mike Brown of Edinburgh University Computing Service, and to Arthur Trew of EPCC, for provision and maintenance of service on the Meiko i860 Computing Surface and the Thinking Machines CM-200. We wish to thank Brian Murdoch for access to a Digital 7640 AXP ("Alpha") system placed at the University of Edinburgh Computing Service by Digital Equipment Corporation for field test.

\section{References}

[1] S.R. Sharpe, Nucl. Phys. B (Proc. Suppl.) 17 (1991) 146

[2] C.R. Allton et al., Nucl. Phys. B (Proc. Suppl.) 20 (1991) 504

[3] A. Abada et al., Nucl. Phys. B376 (1992) 172

[4] C. Bernard, C. Heard, J. Labrenz, A. Soni Nucl. Phys. B (Proc. Suppl.) 26 (1992) 384

[5] C.W. Bernard, J.N. Labrenz and A. Soni, University of Washington Preprint UW/PT93-06 (1993)

[6] J. Adler et al., Phys. Rev. Lett.무 (1988) 1375

[7] E. Eichten, G. Hockney and H.B. Thacker, Nucl. Phys. B (Proc. Suppl.) 17 (1990) 529

[8] C.R. Allton et al., Nucl. Phys. B349 (1991) 598

[9] C. Alexandrou et al., Phys. Lett. B256 (1991) 60

[10] The APE collaboration, C.R. Allton et al., University of Rome Preprint 93/928 (1993)

[11] A. Duncan et al., Nucl. Phys. B(Proc.Suppl.) 30 (1993) 433

[12] C. Alexandrou et al., PSI Preprint PSI-PR-92-27 (1992)

[13] B. Sheikholeslami and R. Wohlert, Nucl. Phys. B259 (1985) 572

[14] G. Heatlie et al., Nucl. Phys. B352 (1991) 266

[15] UKQCD Collaboration, C.R. Allton et al., Edinburgh University Preprint 93/524 (1993)

[16] E. Eichten, Nucl. Phys. B (Proc. Suppl.) 4 (1988) 170 
[17] A. Borrelli and C. Pittori, Nucl. Phys. B385 (1992) 502

[18] A. Borrelli, R. Frezotti, E. Gabrielli and C. Pittori, CERN preprint TH-6587 (1992)

[19] G.P. Lepage and P.B. Mackenzie, Nucl. Phys. B (Proc.Suppl.) 20 (1992) 173; Fermilab preprint PUB-91/355-T(revised) (1992)

[20] P.B.Mackenzie, Nucl. Phys. B (Proc. Suppl.) 30 (1993) 35

[21] G. Martinelli, S.Petrarca, C.T. Sachrajda and A. Vladikas, Phys. Lett. B311 (1993) 241

[22] B. Efron, SIAM Review 21 (1979) 460

[23] UKQCD Collaboration, C.R. Allton et al., Edinburgh University Preprint 92/507 (1992), to be published in Nucl. Phys. $\underline{B}$

[24] Ph. Boucaud et al., Phys. Lett. 220B (1989) 219

[25] UKQCD Collaboration, C.R. Allton et al., Phys. Rev. D47 (1993) 5128

[26] S. Güsken et al., Nucl. Phys. B (Proc. Suppl.) 17 (1990) 361; Phys. Lett. 227B (1989) 266

[27] WA75 Collaboration, S. Aoki et al., CERN preprint PPE/92-157 (1992)

[28] M.B. Voloshin and M.A. Shifman, Yad. Fiz. 47 (1988) 801 [Sov. J. Nucl. Phys. 47 (1988) 511]; H.D. Politzer and M.B.Wise, Phys. Lett. B206 (1988) 681; Phys. Lett. B208 (1988) 504; M. Neubert, Phys. Rev. D46 (1992) 1076; X. Ji and M.J. Musolf, Phys. Lett. B257 (1991) 409; D.J. Broadhurst and A.G. Grozin, Phys. Lett. 274B (1992) 421

[29] UKQCD Collaboration, D.S. Henty and R.D. Kenway, Phys. Lett. B289 (1992) 408

[30] M. Lüscher, Commun. Math. Phys. $\underline{54}$ (1977) 283

[31] K. Symanzik, in Mathematical problems in theoretical physics, ed. R. Schrader, R. Seiler and D.A. Uhlenbrock, Springer Lect. Notes Phys. 153 (1982) 47; Nucl. Phys. B226 (1983) 187, 205

[32] UKQCD Collaboration, J.N. Simone, Nucl. Phys. B (Proc. Suppl.) 30 (1993) 461

[33] B.E. Wilkes, Ph.D. thesis, University of Edinburgh (1993), in preparation 
[34] O.F. Hernandez and B.R. Hill, Phys. Lett. B289 (1992) 417

[35] E. Eichten and B.R. Hill, Phys. Lett. B234 (1990) 511; Phys. Lett. B240 (1990) 193

[36] G. Martinelli, private communication 